\title{
Psychoanalytic and psychodynamic therapies for depression: the evidence base
}

\author{
David Taylor
}

\begin{abstract}
This article argues that the current approach to guideline development for the treatment of depression is not supported by the evidence: clearly depression is not a disease for which treatment efficacy is best determined by short-term randomised controlled trials. As a result, important findings have been marginalised. Different principles of evidence-gathering are described. When a wider range of the available evidence is critically considered the case for dynamic approaches to the treatment of depression can be seen to be stronger than is often thought. Broadly, the benefits of short-term psychodynamic therapies are equivalent in size to the effects of antidepressants and cognitive-behavioural therapy (CBT). The benefits of CBT may occur more quickly, but those of short-term psychodynamic therapies may continue to increase after treatment. There may be a ceiling on the effects of short-term treatments of whatever type. Longer-term psychodynamic treatments may improve associated social, work and personal dysfunctions as well as reductions in depressive symptoms.
\end{abstract}

Latterly, perhaps impelled by the Spirit of the Age as well as by the accelerating cost of medical innovation, governments have increasingly found in evidence-based medicine a necessary instrument, as well as a sensible one. In the UK, the National Institute for Health and Clinical Excellence (NICE) has required its mental health guideline development groups to use a standard, one-size-fits-all approach to evidence pertaining to the various treatments used for specific common mental disorders. When judged by the narrow criterion of the availability of sufficient numbers of high-quality double-blind random allocation controlled trials and meta-analyses, it may be fair to conclude that the evidence base for the most used treatments for depression is weak. However, this kind of null verdict does not take us very far, either in knowledge or in the development of practice. In any case, the restrictive definition of evidence-based medicine in the mental health field which underpins this judgement has some adverse consequences and needs to be heavily qualified.

First, there is a pervasive fallacy that a statement about a 'strong' or a 'weak' evidence base is a statement about the weakness of the treatment rather than about the limitations of the research methods used in the evaluation of that treatment. This introduces damaging confounds. They include the consequences of equating a weak evidence-base with a weak treatment, or a supposedly strong one with a strong treatment. Weak research or flawed guidelines may acquire a status they do not deserve.

Second, although there always may be exceptions, the fundamental difficulties of most people with depression do not resolve with brief interventions of whatever variety. The natural course of depression is often prolonged, relapsing or recurrent (Surtees \& Barkley, 1994). Although patients are significantly helped by any wellinformed and responsive professional intervention, including really good clinical management, the resistant core of any significant psychopathology is a fact of life. To provide a quality mental health service requires care and support, and specific interventions available when needed in long-term packages. The randomised controlled trial (RCT) research agenda has so far been unable to take this fact sufficiently into account.

Third, by and large, the dysfunctions from which patients suffer are not confined to those that can be called a single diagnosis. Most patients not only meet the criteria for several different symptombased diagnoses, but have to cope with many suboptimal functions of the personality (Westen et al, 2004). They complain of doubt of love, of past and present difficulties in terms of personal achievement and work, as well as of existential

David Taylor, a consultant psychotherapist at the Tavistock \& Portman NHS Foundation Trust (120 Belsize Lane, London NW3 5BA, UK. Email: dtaylor@tavi-port.nhs.uk), is the clinical lead of the Tavistock Adult Depression Study (a randomised controlled trial of 60 sessions of weekly psychoanalytic psychotherapy $v$. treatment as usual for patients with chronic, refractory depression). He is a training and supervising psychoanalyst at the Institute of Psychoanalysis. 
and intimate problems of being, identity and so on. By being short-term, RCTs and brief treatment interventions avoid difficulties of this sort, whereas mental health workers should not, and patients cannot, do so.

Thus, for these and other reasons it can be argued that the NICE emphasis on RCTs as the gold standard in the research evaluation of psychological therapy outcome is as unfounded as its evidence base is weak.

The position seems to be that different theoretical approaches, such as the cognitive, the pharmacological, the psychiatric, or the psychoanalytic, the clinical or the 'scientistic', have very different ideas about the principles that should underpin the methodology of outcome research and of evidence-based guidelines. These are serious and important disagreements about what counts as quality in evidence and fact, and about the place of clinical knowledge in the discourse. These different positions translate into the way questions about efficacy and effectiveness are asked and the way in which they are answered. They give rise to serious problems of incommensurability: when we ask questions about complex issues we must of necessity choose one among several approaches to frame our questions and to provide the concepts and terminologies we think are best suited to define, collect, observe and interpret what we count as empirical data. Empirical data are, in truth, empirical only up to a point. Beyond that point they are highly method-dependent. We cannot simply examine the facts obtained by one method with the concepts belonging to another. To cap it all, the factors that determine which of the above approaches is dominant involve deepseated personal preferences that are hard waxed against discrepant findings or arguments.

This article is in three sections. The first outlines the preliminaries necessary to provide a frame of reference for the review of evidence that follows. This evidence base is often an inconclusive and contradictory set of findings from treatment outcome studies relevant to depression. Often it seems more like a quagmire than firm ground. I focus here on the evidence pertaining to psychoanalytic/ psychodynamic approaches. I hope that it will be possible to begin to see how, if we judge by lights other than those subscribing to the primacy of method, the evidence base for psychodynamic approaches is not actually that weak. There is, in fact, a web of evidence supporting their value. Indeed, because this extends into so many domains it has not been possible to give more than a hint of the wide range of different types of evidence that have a bearing on this issue. In the final section I discuss this evaluation and suggest some conclusions.

\section{Preliminaries for a frame of reference}

\section{Prevalence, course and nature of depression}

In high-income countries, over the course of a year about $6 \%$ of the adult population will suffer a major depressive disorder, and $6 \%$ will experience dysthymia, with the lifetime prevalence of the disorder being about 17\% (Weissman et al, 1991). In the community, between 20 and $30 \%$ of untreated depressed individuals will still be depressed a year later (Sargeant et al, 1990). Put the other way round, $70 \%$ of people with untreated depression will recover without intervention in the course of a year. Generally, the risk of further episodes over the 10 to 15 years after an index episode is more than $85 \%$ (Mueller et al, 1999). Of those who initially respond to medication, $30 \%$ will relapse within a year, rising to as many as $75 \%$ after 5 years (Hirschfeld, 1991). Each person with major depressive disorder will experience on average four episodes during their lifetime. Each of these episodes will last an average of 20 weeks with ongoing symptoms and disability during the periods of 'remission' (Judd, 1997; Judd et al, 1997).

As these figures suggest, depression can be a circumscribed, self-limiting condition for some people, but a significant proportion will suffer a pattern of remission and relapse (Surtees \& Barkley, 1994), and will show a tendency for a deteriorating outlook as time goes by (Paykel, 1994) (Box 1). Yet, the majority of RCTs of psychological therapy and pharmacotherapy involve short-term treatments of acute-phase depression, with non-existent or brief follow-ups. These studies therefore do not provide reliable information about efficacy over

\section{Box 1 The natural course of depression}

- For about $50 \%$ of individuals, the duration of illness is 3 months; thereafter the probability of remission diminishes rapidly

- $60 \%$ of people receiving antidepressant medication still meet the criteria for major depressive disorder after 1 year of treatment, and $20 \%$ after 2 years

- Up to $90 \%$ of patients develop new episodes after recovery

- Only $20 \%$ of people hospitalised for depression remained well over a 15-year follow-up

(Lee \& Murray, 1988; Coryell et al, 1994; Goldberg et al, 1998; Spijker et al, 2002) 
Box 2 Problems with single-disorder research and treatment guidelines

- Apparently exact rates of diagnosis lend pseudo-objectivity to the familiar taxonomy

- Results depend on which of several diagnostic schemes is used (Goldberg \& Goodyer, 2005)

- A minority of patients satisfy the criteria of only one diagnosis. Patients meeting criteria for major depressive disorder are nine times more likely than chance to meet the criteria for other conditions (Angst \& Dobler-Mikola, 1984, 1985)

- $57.5 \%$ of patients with depression also have anxiety disorders (Kessler et al, 2003)

- $50-90 \%$ of patients with Axis I conditions also meet the criteria for other Axis I or Axis II disorders (Westen et al, 2004)

- Index presentations commonly change over time, with the features of depression, anxiety, phobic or obsessive-compulsive disorders now uppermost, now unobtrusive

- Patientscomplain of problems of functioning and of life not captured by measures of symptoms or categorical diagnoses

the medium and longer term, especially in relation to those who have more chronic or severe forms of depression.

\section{The taxonomy of depression}

The data above were arrived at using diagnostic schemes based on the idea that depression, generalised anxiety disorder and obsessive-compulsive disorder are distinct entities. If they truly were separate entities, they would be expected to occur independently of each other, to have their own particular aetiology, and each would respond to its own specific treatment acting on pathological processes specific to the disorder. What we find does not support this idea (Box 2).

\section{The psychoanalyticlpsychodynamic model of therapy}

The term 'psychodynamic psychotherapy' encompasses therapeutic approaches derived originally from psychoanalysis and depth psychology. Although psychodynamic psychotherapies are various, the role of early development, unconscious processes and the transference significance of the therapeutic relationship are to some extent common to most.

In classical psychoanalysis the analyst seeks to follow the patient rather than to set or impose an agenda. Psychoanalysts try to understand a unique process set in motion by the patient's preoccupations, anxieties, difficulties, character and, above all, by his or her way of relating. Exploring sensitive personal areas requires a stable, sympathetic yet objective discipline. The approach is based on a view that everyone is subject to a flux of internal conditions, which to some extent are always going to be difficult for the individual to deal with or fully acknowledge. The psychoanalyst's theoretical knowledge and personal analysis provides a detailed familiarity with the nature of these internal states and the powerful feelings associated with primary relationships in the child and the adult.

In psychoanalysis, the patient uses the couch, has sessions five times a week, and the treatment lasts for some years. Although in some ways it can be likened as much to education as to a form of treatment, in significant respects it differs from both. Like any significant piece of learning, psychoanalytic change occurs slowly but the emotional and cognitive developments that are involved are based on learning by internalisation rather than learning by rote, or memorising. This internalisation process involves the patient in a sustained contact with previously under-explored areas of their personality as they function within the specialised relationship between patient and analyst (Milton, 2001). Analysts use their knowledge and training to be able to see into, to challenge, and to contain and to tolerate the areas of difficulty the patient brings. As a result, the patient gradually takes in a capacity to feel more comfortable with these and other emotionally difficult areas.

Psychoanalysiscanbeengrossingand fascinating. Because of its meaningfulness many patients feel much supported, partly because of its openness to emotionally demanding or turbulent areas. This explains why many people will make considerable sacrifices to have an analysis. However, it is not to everyone's taste or liking. Psychoanalysis has the capacity to evoke great hatred and contempt as well as great appreciation and gratitude.

To make the value of psychoanalytic thinking available to patients for whom a full analysis is either impossible for practical reasons, or not indicated for clinical ones, many shorter or less intensive forms of individual and group psychotherapy have been devised. The general psychoanalytic models that underpin these applications remain non-directive and are offered in short-, mediumand longer-term formats. Centres of psychotherapy 
in the UK offer a model of once-weekly psychoanalytic psychotherapy over 1 year (45 sessions), or occasionally a little longer. The social insurance funded healthcare systems of a number of European countries routinely fund up to 300 sessions of twice- or thrice-weekly psychoanalytic psychotherapy sessions.

Over the years a number of more structured, and usually short, forms of psychodynamic therapy have also been developed. Typically, these include actively formulating a focus on the particular unconscious operations and emotions thought to underlie the patient's symptoms and complaints. The model of Davanloo (1980), for instance, is organised around the idea of a triangle of conflict (feelings, anxiety and defence) and a triangle of person (past, therapist and current) to frame interventions. The brief psychotherapy model of Malan (1976) is similar. However, although these structured approaches are more concerned with achieving specific focal aims, patients are still offered and still obtain insight. They are not given homework or didactically taught mental skills such as thought-monitoring and re-framing.

\section{A brief account of the psychoanalytic understanding of depression}

Psychoanalysis regards mourning and depression as types of bio-psychological reactions to loss (Box 3). Any significant loss stimulates fear, anxiety and anger. The activity of the emotional system connected with fight or flight is triggered and emotional systems to do with nurturance are enormously disturbed. These powerful emotional reactions have to be managed by the organism. Freud (1917) compared the mental processes involved in mourning with those in depression. The ability to mourn is an adaptive capacity involving relinquishment. It can lead to mental growth and the return of the capacity to feel love at a new level. Relinquishment is more difficult for those susceptible to depression, because of underlying difficulties with tolerating the fundamental separateness of the loved object. Such individuals are unable to cope with the love and the hate that arise in connection with absence while at the same time feeling to some degree secure. Their common origin in loss means that these two major states of mind can coexist and also interact with each other in very significant ways.

Mourning the lost object is one of the ways it is possible to recover from depression. This is what is happening over the course of the self-limiting depressions that so commonly occur at the turningpoints of life. In mourning, individuals remain
Box 3 Some elements of the psychoanalytic theory of depression

- Excessive neediness, dependence and ambivalence in love relationships predispose to depression after object loss

- Earlier developmental difficulties with object loss are continuously re-evoked in the course of later relationships of work and love. Just as 'every finding of an object is a re-finding', every loss revives an earlier one (Freud, 1905; Klein, 1940)

- Early feeding and affective bond experiences are central to nurturance and fight-or-flight systems. They are of particular importance in a vulnerability to depressive disorders in adulthood

- An excessively severe or envious superego (Freud, 1917) influences the various levels of severity, chronicity and refractoriness that depression can take

- Both environmental and constitutional (genetic) factors play a part

consciously preoccupied with whom or with what has been lost, but in deep depression people often no longer know why they are depressed. In mourning it is the world that is felt to have lost meaning but in depression it is the self that is viewed as diminished or bad. The self undergoes this alteration through its becoming identified with the lost object. Identification is an unconscious mechanism which involves 'becoming the same as'. In depression, identification occurs on the basis of sympathy with the object, sometimes out of guilt or sometimes as a way of keeping the relationship with the lost person present-within rather than absent-without.

The explanation of these differences between mourning and depression rests on the finding that the acceptance of the loss is more difficult in depression (Klein, 1940). Although we would be faithless and shallow if we lacked all tenacity in our relations with the world, in those with a vulnerability to depression the possibility that the tie to the object might be loosened feels not possible. There tends to be too much ambivalence and insecurity in relation to the love object to allow for this. There is a conflict between feelings of love and antagonism. Also, a desperate effort to spare the loved object from aggression lends intense painfulness to the internal struggles of the person who is prone to depression. This 'sparing' is a sine qua non of depression and is its ethical dimension. Because aggression cannot simply be wished out of 
existence, the attempt to spare the object inevitably means that the hostility must be turned inward. This is how people who are depressed come to believe that they are bad, disliked, hated or hateful. Many of the somatic and vegetative symptoms of depression arise from inhibited feelings of arousal, hostility and fear operating at the somatic level as well as within the more familiar symbolic and representational levels of feeling and thought.

This turning of aggression and anxiety against the self is mediated by an internal structure, the critical agency, the superego. The superego is coloured by the individual's own hostile and envious feelings so that, other things being equal, the more an individual controls his or her aggression towards others the more severe their superego becomes.

Although ultimately our dispositions are the outcome of gene-environment interactions of enormous complexity, the emotional work we are able to do with the troubles of our own minds can, over the course of time, make a big difference to our final outcome.

\section{The outcome evidence for psychoanalytic/psychodynamic treatments of depression}

The observations summarised in Box 2 support the view of many clinicians that the current way of classifying and analysing data dismembers otherwise instructive relationships that exist between the different types of symptom found in patients with a broader category of 'common mental disorder'. However, the idea of distinct, homogeneous mental 'diseases' has dominated research and guideline development (including those of NICE) in the past 25 years, exerting great influence on the type of study done and the kind of results available. (For a good general review of the evidence for psychodynamic therapies see Fonagy et al, 2005.) What follows will therefore include the results of some meta-analyses and trials that have used the concept of common mental disorders as a way of describing their study populations.

\section{Short-term psychodynamic psychotherapy}

In line with general usage, the various shorter-term formats, whether structured or non-directive, will be referred to collectively as short-term psychodynamic psychotherapy (STPP). Most of the RCTs examining the efficacy of psychoanalytic or psychodynamic approaches have studied forms of STPP. Usually this means no more than 20 sessions.
Efficacy of psychological therapy in the treatment of generic disorders

In a mother of all meta-analyses, a meta-analysis of 45 meta-analyses of the results of a wide variety of brief psychological, educational and behavioural treatment trials for an equally wide range of nonpsychotic disorders, Lipsey \& Wilson (1993) came up with an estimate of the efficacy of psychological interventions as a 'generic' type. They found an effect size of +0.76 for all forms of psychological therapy when compared with waiting-list or minimaltreatment control conditions. This indicates that the post-treatment symptoms of those who receive any form of brief psychological therapy will be about three-quarters of a standard deviation better than that of those who do not. This is a worthwhile benefit, but under the limited parameters of brief therapy research it has been hard to demonstrate a clear magnitude of difference between this size of effect and that associated with placebo or 'treatment as usual' (TAU), especially should the treatment be of a good standard.

The reader will come to see how, in spite of numerous efforts to pick it apart, a persistent thread of non-specificity runs through the findings of psychological therapy research trials. This socalled 'equivalence paradox' refers to how difficult it has proved for outcome research to demonstrate specific differences, whether according to degree of effect, diagnosis or type of psychological therapy. For this reason, experienced psychotherapy researchers summarise the previous decades of research in their field with conclusions such as, 'the generic efficacy of psychotherapy compared to no-treatment has been established for a long time ... from mildly disturbed persons with specific limited symptoms as well as from severely impaired patients ... study after study, metaanalysis after meta-analysis, have empirically validated psychotherapy as a treatment' (Lambert, 1992: p. 97). Luborsky et al in 1975 applied the term 'Dodo bird effect' to the way every research camp in psychological therapy would present data that prove that its favourite is the best, while the rival is perfidious. The description refers to Lewis Carroll's Alice in Wonderland, in which the Dodo silences the bitter squabbles at the end of a race by announcing that 'everybody has won and all must have prizes'. The stability of Luborsky et al's conclusion was more recently confirmed by Wampold et al (1997).

Efficacy of generic psychological therapies in the treatment of depression

Robinsonetal (1990), in their admirably lucid review of 58 controlled studies of the use of psychotherapy with patients with a formal diagnosis of depression, 
found effect sizes of 0.73 post-treatment and 0.68 at follow-up. These are of the same order as those quoted above for a much wider group of disorders. Their figures indicated that collectively these different types of psychological therapy offer a moderate benefit in the treatment of depression compared with no treatment. Although the authors' initial analysis found the effect sizes for cognitive, behavioural and cognitive-behavioural therapy (CBT) to be about twice those of 'general verbal therapies' (a category that included STPP), they found that this difference disappeared when the treatment allegiance of the researcher and the quality of the trial were taken into account. Their ultimate finding was that there was 'no reliable difference' between the efficacies of different forms of therapy in the treatment of depression. Each form was making roughly equal contributions to the final overall effect.

\section{Efficacy of STPP compared with no or minimal treatment for diagnosed depression}

The Cochrane review by Abbass et al (2006) found only two RCTs of sufficient quality giving data on the more exact question of the efficacy of STPP $v$. 'no treatment' or TAU in patients with a formal diagnosis of depression. This was out of a total of 23 RCTs examining STPP in the treatment of common mental disorders. In an earlier review, Leichsenring et al (2004) had found a third study. The three studies were as follows.

De Jonghe et al (2004) In the treatment of adults with mild to moderate major depressive disorder $(75 \%$ having an illness of less than 2 years' duration), the authors compared a form of STPP (maximum of 16 sessions) alone with STPP and antidepressant medication. Although there were marked differences in their acceptability to patients, the efficacy of both formats in terms of effect size, imputed on the basis of the difference between baseline measures and measures at 24 weeks in the two groups, was large $(\geq 0.8)$ and more or less equivalent. Note how similar this is to the value reported by Lipsey \& Wilson (1993). This study is considered further below.

Thompson et al (1987) In the treatment of major depressive disorder in the elderly the authors compared 16-20 sessions of STPP with an equivalent number of CBT and behavioural therapy sessions and a waiting-list control state. By the end of 6 weeks, patients in the treatment conditions showed improvement of the familiar order, whereas controls did not. The results of this study also have a bearing on the comparative efficacy of the different forms of psychotherapy (see below).
Cooper et al (2003) In the treatment of postnatal depression, the authors investigated the efficacy of three forms of brief intervention, one of which was 10 sessions of psychodynamic therapy, compared with routine primary care, in post-partum women meeting the criteria for major depressive disorder. At $4 \frac{1}{2}$ months, $70 \%$ of the psychodynamic cohort were judged to be recovered, compared with $40 \%$ of the control condition. By the 9-month follow-up, however, the benefit of treatment over usual care was no longer evident. Moreover, the three forms of therapy did not reduce the incidence of further episodes of postnatal depression. Psychodynamic psychotherapy did, however, seem to accelerate recovery, which in the majority of cases would have occurred but more slowly. This study had a long 5-year follow-up, unlike most RCTs.

\section{Efficacy of STPP compared with no or minimal treatment for common mental disorders}

According to psychoanalytic theory depression, anxiety, obsessional features, personality problems and a variety of more subtle manifestations are thought to be interconnected in a dynamic way. Like an increasing number of psychiatrists, psychoanalytically informed clinicians and researchers have serious reservations about the validity of using the single-diagnosis concept of depression in outcome trials. Many have argued that it makes more sense to study the effects of treatment in patient samples that include mixed or combined disorders. The search criteria used in single-disorder guidelines such as those of NICE have tended to exclude the findings of studies of this sort.

Leichsenring et al (2004) This review found seven RCTs of sufficient quality which had examined STPP against no treatment or TAU for a mixture of common mental disorders. Shortterm psychodynamic psychotherapy was found to be 'significantly superior' to these placebo conditions, with effect sizes ranging from 0.59 to 1.17 depending on the domain measured (target problem, general psychiatric symptoms and social functioning) and the measure used. 'No treatment' conditions were found to have 'before' and 'after' effect sizes, which ranged from 0.12 to 0.27 , while TAU was found to be capable of more substantial benefits, ranging from 0.22 to 0.95 , depending presumably on the intensity, adequacy and quality of the TAU provided.

Abbass et al (2006) This review of 23 RCTs examined the efficacy of STPP relative to no treatment or TAU in the category of common mental disorder. 
They pooled patient samples which had originally been selected on the basis of several specific common disorder diagnoses. In addition to depression, these included somatoform disorders (irritable bowel syndrome, chronic pain, etc.), anxiety and personality disorders and the heterogeneous mixture of disorders found in a non-responding sample of non-psychotic general psychiatric outpatients. Some of the studies were of samples of patients with chronic, difficult-to-treat disorders and comorbid substance misuse. Only 8 overlapped with Leichsenring's et al's review. Combined, the 23 trials involved 1431 patients. Results indicated that STPP (for a mean of 15 sessions) produced modest to moderate gains across a wide range of symptom categories for a wide variety of patients. Furthermore, these gains were often sustained or had increased at longer-term follow-up.

Svartberg \& Stiles (1991), Crits-Christoph (1992), Anderson \& Lambert (1995) For mixed disorders, these three meta-analyses of all found STPP to be superior to no treatment or minimal treatment at both short- and longer-term follow-ups.

Efficacy of STPP compared with no or minimal treatment for depressive symptoms in common mental disorders

The composite, common mental disorder group assembled in the reviews of Leichsenring (2001) and Abbass et al (2006) included many patients with significant depressive symptomatology across the diagnostic categories. Of the 23 studies in Abbass's review, 14 had employed measures of depression. In terms of relief from depressive symptoms these showed in the short term ( $\leq 3$ months, 11 studies) a standardised mean difference (SMD) of 0.59 , in the medium term (3-9 months, 5 studies) an SMD of 0.41 , and in the longer term ( $\geq 9$ months, 6 studies) an SMD of 0.98 relative to controls. These amount to moderate treatment effects and, in line with trend, the longer-term follow-up finding is more substantial.

Efficacy of STPP compared with other forms of psychological therapy

The meta-analyses of Crits-Christoph (1992) and Anderson \& Lambert (1995) similarly found STPP to be equal in efficacy to other forms of psychological therapy, as did the Leichsenring et al (2004) study. Only one meta-analysis, that of Svartberg \& Stiles (1991) found STPP to be less effective than other short-term treatments such as CBT. However, when controls for quality were introduced, this putative superiority evaporated.
Efficacy of STPP compared with CBT

and behavioural therapy for depression

Churchill's et al's (2001) systematic review of controlled trials of brief psychological treatments for depression, part of the NHS Health Technology Assessment programme, found six studies (five randomised controlled and one controlled clinical trial) comparing STPP with CBT in formats of less than 20 sessions.

Pooling dichotomous recovery/non-recovery data suggested that, by the end of the treatment, the odds of recovery for patients receiving CBT were at least twice those for patients receiving STPP.

However, the reviewers noted the possibility of a bias towards the CBT condition in these trials. More than half the studies were conducted by researchers with a CBT preference; these trials had used psychodynamic therapy only as a secondary comparison condition. Again, when ratings for the quality of the trial and the quality of treatment delivery were included, this marked difference disappeared.

The difference reported between CBT and brief psychodynamic treatments also disappeared when follow-up data were reported. In two of the studies this was at 3 months, and in a third at 1 year. The remainder did not report follow-up data.

Leichsenring (2001) came to similar conclusions. He confirmed that STPP was associated with statistically significant reductions in depressive symptoms before and after treatment. Out of a total of 60 possible comparisons between STPP and CBT he found no difference in 58 , and a small effect in favour of CBT in 2. He calculated the mean 'before and after' success rates of STPP and CBT as $46 \%$ and $54 \%$ respectively - equivalent to a small effect size in favour of CBT. Wampold et al (2002) reported similar findings.

\section{Efficacy of psychotherapy compared with antidepressants}

According to Roth \& Fonagy (2004), when psychotherapy and medication are offered alone they seem to be of roughly equivalent efficacy. Most, but not all, of this evidence is from interpersonal therapy and CBT studies. The earlier review of Robinson et al (1990) found 15 studies examining this question, including a few looking at the effect of combining psychological therapy with antidepressants. Eight of these 15 studies were of CBT, three were of behavioural therapy and four tested what the authors describe as 'general verbal' therapy, which includes dynamic approaches.

The studies yielded a small effect size in favour of psychological therapy, but this may be an artefact. Once again, when allegiance effects (Luborsky 
et al, 1999) were taken into account no reliable differences could be found between psychological therapy and pharmacotherapy. Publication bias and selective reporting are now known to have influenced findings bearing on this question.

However, there is some evidence (Thase et al, 1997) that antidepressants are more efficacious for people with more severe depression than for those with depression of mild to moderate severity, or those for whom complex psychosocial factors of history and personality are significant parts of the presentation.

\section{Efficacy of combined treatments}

Augmenting STPP with antidepressants (v. STPP alone) De Jonghe et al (2004) examined the issue of whether the addition of antidepressants to 16 sessions of STPP would improve on the outcome of major depression of mild to moderate severity treated with STPP alone. A sample of about 200 psychiatric out-patients with major depressive disorder was treated and followed up over a 6-month period.

Both treatment packages produced broadly equivalent positive effects. The success rate in the STPP-alone arm was $73 \%$ as against $81 \%$ in the combined treatment arm, with pre-post effect sizes on the Hamilton Rating Scale for Depression (HRSD) of 1.22 and 1.53 respectively. By 24 weeks, HRSD ratings had fallen from an initial average of 18 to one of 10 . The comparison findings indicated a small benefit in favour of combining antidepressants with STPP, but these reached significance only in respect of the patient's selfreport measure (with a medium effect size of 0.49 ). Because there was no drug placebo control in the trial the mechanism of this effect is uncertain.

Other important findings concerned the strength of patients' attitudes to the two kinds of treatment. Although $25 \%$ of psychotherapy patients broke off their treatment, psychotherapy still seemed to be more acceptable. More patients refused to enter the pharmacotherapy arm in the first place, and by 6 months a further $35 \%$ had withdrawn from it.

Augmenting antidepressants with STPP (v. antidepressants alone) Burnand et al (2002) investigated the question of combined therapy the other way around: augmenting antidepressant medication with a 10-session form of STPP, administered in this case by well-trained nurses, to enhance the outcomes of patients in an acute-phase major depressive episode. The patients had been referred to an out-patient psychiatric service, and had moderate to severe degrees of depression; half had had previous episodes; half had some form of personality disorder. They were as a whole slightly more ill than the de Jonghe et al (2004) sample. The final sample analysed consisted of 74 patients randomised to the two conditions - antidepressants (mainly clomipramine) combined with supportive time with a designated key worker $v$. antidepressants plus 10 sessions of STPP with a trained nurse-therapist.

After the 10-week treatment period the mean HRSD scores in both groups had reduced from about 24 to about $9(P>0.001)$ but with little difference between the comparison conditions on this measure.

However, the combination of STPP and antidepressants was better than antidepressants alone on several other important indices: patients who received STPP were significantly less likely to still meet the criteria for major depressive disorder after 10 weeks $(9 \%)$ than those who had not $(28 \%)$.

The combination group needed significantly fewer days off work (46 v. 57), and they had both a lower rate and fewer days of hospitalisation. Cost-benefit comparisons showed that STPP plus antidepressants had resulted in savings of \$2311 per patient over the 10-week period. For patients in full employment before they became ill savings were greater (\$3394).

Once it is recollected that depression can be a long-term relapsing condition, the drawbacks of the trials summarised above are hard to deny in respect of all types of treatment. They are nearly all concerned only with the short-term outcome of brief treatments. The findings of trials of this kind also suggest a ceiling on the effects of brief therapies of whatever type. To go beyond these limitations it is important to look to studies of longer-term treatments of patients with chronic or harder-to-treat depression of one kind or another. Furthermore, because the dysfunctions associated with depression extend beyond what symptom measures capture, we need to develop our understanding of these and to find ways of evaluating change.

\section{Efficacy of treatments of chronic or treatment- refractory depression}

Stimpson et al (2002) found 17 RCTs which examined various pharmacological approaches to the treatment of patients with unipolar depression who had not responded to a minimum of 4 weeks of antidepressant treatment.

On the basis of these 17 studies, they concluded that there was little in the way of evidence to guide the treatment of patients who had not responded to a first course of antidepressants. Within their criteria, they found no satisfactory trials of psychological therapy for chronic or treatmentresistant depression. 
By using more lax inclusion criteria, McPherson et al (2005) did find a few studies of psychological treatments, including single case studies. These mainly used CBT. Although they mostly showed reduction in symptoms, they all had short followup times and very small numbers. Interestingly, and presumably in response to the severity of the psychopathology, the CBT treatments involved seemed to be showing a marked tendency to become longer than the short 10 or 20 sessions that have been a principle selling point of the method. In one small study the eight participants had 39 sessions over 8 months - beginning to approach something like a medium-length psychodynamic treatment!

Two RCTs are currently under way with this patient group. The Tavistock Adult Depression Study (Publication ID M0001169680 at http:// www.nihr.ac.uk) is examining the efficacy of medium-term (60 sessions) weekly psychoanalytic psychotherapy $v$. TAU in 90 patients with treatmentresistant depression. Eligibility criteria include at least two failed treatments as well as a diagnosis of major depressive disorder. The average duration of illness is 10-15 years and most patients have been found also to meet the criteria for at least one DSM Axis II disorder. The trial is due to report in 2010. A similar multicentre German RCT combined with a naturalistic arm began last year. The Langzeittherapie bei chronischen Depressionen (LAC) study, led by Professor M. Leuzinger-Bohleber, is comparing a longer psychoanalytic psychotherapy treatment with CBT and has an intended sample of 240 (http://www.sfi-frankfurt.de/forschung/ forschungsfeld-2/depressionsstudie.html).

Efficacy of long-term $v$. short-term psychodynamic/psychoanalytic therapies in depression and common mental disorders

The Helsinki Psychotherapy Study Group's RCT (Knekt et al, 2008) has compared the effectiveness of two forms of short-term therapy with a longterm psychodynamic psychotherapy. So far followup data over 3 years have been reported. The study also has an arm for full psychoanalysis, which is about to report along with further follow-up data. The two short-term treatments were a problemsolving solution (solution-focused therapy) (about 10 sessions, including family work over 8 months; Lambert et al, 1998) and a form of STPP based on the Malan model (20 sessions over 6 months). The long-term treatment involved 2-3 sessions of psychodynamic therapy a week over 3 years (average number of sessions was 232). The 326 participants, between 20 and 45 years of age, had been referred and treated as out-patients for mild to moderate DSM-IV mood or anxiety disorders for at least 1 year; $68 \%$ met the criteria for major depressive disorder, $44 \%$ met those for generalised anxiety disorder, $18 \%$ met those for personality disorder, and $9 \%$ had made a suicide attempt.

All three interventions were associated with significant reductions on all symptom measures (depression, anxiety and general). Over the 3-year period the average reduction in Beck Depression Inventory (BDI) score was 51\% (with effect sizes ranging from 0.87 to 1.52 ). At entry the mean $\mathrm{BDI}$ score was 18; in those given the two short-term treatments this had come down to about 10, and in those who had had long-term therapy it was about 7. These benefits are comparable to those found in studies involving CBT.

Participants who received the short-term treatments typically showed an early response which reached an upper limit by 12 months. These gains proved lasting over the entire 3-year follow-up.

In contrast, the long-term therapy group initially lagged behind, showing significantly less improvement over the first year. However, the gains in this group gradually increased so that by the 3-year point on all measures their symptoms were less than those of the short-term treatment groups; with respect to anxiety they were four times more likely to be recovered, but with respect to the depression measures the difference was not of the same degree. This pattern has also been found in other studies (Kopta et al, 1994).

Overall, about $20 \%$ of those who initially met criteria for personality disorder no longer did at 7 months. In the STPP group there was a continued reduction in the features of personality disorder, so that by 12 months only $9 \%$ met the criteria. This continued improvement in personality functioning did not occur in the solution-focused therapy group.

Evidence from retrospective and prospective longitudinal effectiveness studies

Psychological therapies require the patients' personal choice, voluntary mental work and engagement.Especiallyoverthemediumandlonger term, RCTs, with their analogies to medication trials, do not fit easily with the requirements of human relatedness. Practically and ethically it is not possible to randomly allocate over long durations. It is entirely unrealistic to require RCT evidence for the outcome of these longer therapies. Instead, the evidence from long-term and, until recently, usually retrospective effectiveness studies needs to be taken more seriously.

A carefully designed naturalistic long-term follow-up in Germany of 402 patients (of whom 128 had affective disturbances) who had received psychoanalysis and long-term psychoanalytic 
psychotherapy (Beutel \& Rasting, 2002) showed that a clinically representative group of patients with significant depressive symptoms had moved into the normal range of scores. At long-term follow-up, this group was doing better in terms of days off work than the population norm. The study included in-depth qualitative interviews that made it possible to discern distinct and differing patterns of change in the way that various personality types managed their thoughts and feelings. These patterns included the emergence of reflective functioning.

Other studies have confirmed the clinical impression that improvement may continue after psychodynamic or psychoanalytic psychotherapy ends. The Stockholm Outcome of Psychoanalysis Project, for example, has demonstrated that patients gained in strength and capacity after treatment had ended (Sandell et al, 2000; Blomberg et al, 2001).

\section{Discussion and conclusions}

\section{Do short-term treatments cure depression?}

Robinson et al (1990) enquired whether the clinical effects of short-term psychological and pharmacological treatments amount to a cure of depression. In their sample of studies the mean pre-treatment BDI score was 21.8 and post-treatment this had fallen to 11.8. The corresponding figures for untreated controls were 20.7 and 18.1. The mean BDI score of unselected samples from the general population is 7.0. For the fraction of the general population that is contented this comes down to 4.9. Thus, these treatment effects move patients to within 1 standard deviation of the general population mean, and to within 1.5 standard deviations of the contented among us. These are not trivial effects but even by the restricted sensitivity of the BDI they suggest that on a good day the average welltreated recipient of short-term treatments is still more depressed than most of us on a bad day, and is even further from the ideal of normative functioning. The consistency of these estimates points towards there being a ceiling to what can be achieved with short-term treatments.

\section{Does the evidence base need to remain an industry of small differences?}

It is still possible to sum up the current state of evidence with the following blanket conclusion: antidepressant medication and the different varieties of short-term psychological therapy are capable of generating an improvement of about
$12-13$ points on the BDI, and there is not much to choose between them. Also, as clearly indicated by the findings of the Robins \& Regier (1991) study, the 'placebo' effect in trials of treatments for depression can be consistently large, amounting to an average of 10 points' improvement.

Viewed in this way, it is understandable that many clinicians throw up their hands at a research base that seems more like a heap of dust. It does seem that in respect of treatment research into depression (and other common mental disorders) the Dodobird'sjudgement, at least, is alive and well. It lives on in an evolutionary backwater maintained by three factors: the conviction that short-term RCTs are guardians of truth, the continued use of instruments that are insensitive to the many aspects of patients' dysfunctionality that lie outside symptom frames of reference, and a homoeopathic fantasy that brief psychological treatments are highly potent. If these items stay as they are, the difficulty of demonstrating informative differences between therapies will continue until the wishful side has overwhelmed realism, probably through sheer weight of numbers.

Within the remit of this article it has only been possible to present the evidence from outcome trials, and that in a summarised, incomplete way. However, when we questioned the inclusion and exclusion criteria of guidelines, and the effect of continuing to use specific psychiatric diagnoses as an organising principle, we found that relevant evidence had got left out. Moreover, when we allow clinical experience a little more authority over short-term outcome research trials, other points come into view. If we proceed to admit evidence from other kinds of science, a yet more interesting range of conclusions becomes possible. Epidemiology, observations of natural course, developmental psychopathology, studies of psychosocial factors, life-events, experimental studies and psychoanalytic findings themselves know a great deal about the nature and origins of depression and, in passing, provide supporting evidence for psychoanalytic theories about depression.

Although this more sophisticated conception of the evidence base cannot tell us directly which treatments will prove effective, it does enable us to know the parameters of the disorder and the limits on the plasticity of individuals. These are essential guides to the potential of different treatment. They are scales against which to measure some of the wilder claims. Psychiatry could go further and reintroduce other ideas, such as 'profound' v. 'banal', to counter the narrowness of the sometimes pseudostatistical significance/non-significance formula. 


\section{Conclusions}

This wider view of evidence is consistent with the following.

- Effect sizes for STPP similar to those for other therapies and, like them, superior to minimal treatment control conditions. The NICE depression guideline's solitary recommendation for psychodynamic approaches is 'Psychodynamic psychotherapy may be considered for the treatment of the complex comorbidities that may be present along with depression' (National Collaborating Centre for Mental Health, 2004: p. 63). In the light of the wider evidence available this seems nugatory, especially when it is recollected that comorbidity is more the rule than the exception. The guideline is under revision.

- A tendency for the effect of CBT to come into play more quickly than that of STPP, but with longer follow-ups the differential effect that arises from this delay disappears (Shapiro et al, 1994).

- Longer-term psychodynamic and psychoanalytic therapies, by addressing the dysfunctions of persons, may have the potential to go above an efficacy ceiling that operates in short-term treatments. There are indications that patients in longer-term psychoanalytic treatments begin to manifest qualitatively different types of change, which can move them further into the normal range.

- Although results from studies of combined drug and psychological therapy may be mixed, there is evidence of 'extra value' from adding STPP to medication. Also, adding medication to STPP, especially where vegetative symptoms are pronounced, seems entirely sensible. There seem to be no trials addressing benefits from sequences of CBT $\rightarrow$ STPP or STPP $\rightarrow$ CBT.

- The reasons patients have for preferring one treatment over another, including one form of psychological therapy rather than another, have been under-explored.

- The value of qualitative in-depth studies of single or small numbers of cases and of more naturalistic designs has been underestimated.

- We should think a lot more about the placebo effect and the disputes over valuable but limited gains. The psychological mechanisms involved in the placebo responsein depression have a role in normal emotional life. The belief in good medicines dispensed by good people who can provide succour is one of the features of normal mental functioning that fails in depression. The restoration of this belief-function may be important for recovery in depression.

All of these considerations are pointers towards optimal or enhanced services for patients suffering from the complex range of disorders that goes under the general rubric of depression.

\section{Declaration of interest}

D.T. is the clinical lead of the Tavistock Adult Depression Study, an RCT evaluating 18 months of weekly psychoanalytic psychotherapy in the treatment of chronic, treatment-resistant depression.

\section{References}

Abbass, A. A., Hancock, J. T., Henderson, J., et al (2006) Shortterm psychodynamic psychotherapies for common mental disorders. Cochrane Database of Systematic Reviews, issue 4, CD004687.

Anderson, E. M. \& Lambert, M. J. (1995) Short-term dynamically oriented psychotherapy: a review and meta-analysis. Clinical Psychology Review, 15, 503-514.

Angst, J. \& Dobler-Mikola, A. (1984) The Zurich Study II. The continuum from depressive to pathological mood swings. European Archives of Psychiatry and Neurological Science, 234, 21-29.

Angst, J. \& Dobler-Mikola, A. (1985) The Zurich Study. VI. A continuum from depression to anxiety disorders? European Archives of Psychiatry and Neurological Science, 235, 179-186.

Beutel, M. \& Rasting, M. (2002) Long-term treatments from the perspectives of the former patients. In The Outcomes of Psychoanalytic Treatment (eds M. Leuzinger-Bohleber \& M. Target), ch. 11. Whurr.

Blomberg, J., Lazar, A. \& Sandell, R. (2001) Outcome of patients in long-term psychoanalytical treatments. First findings of the Stockholm Outcome of Psychotherapy and Psychoanalysis (STOPP) study. Psychotherapy Research, 11, 361-382.

Burnand, Y., Andreoli, A., Kolatte, E., et al (2002) Psychodynamic psychotherapy and clomipramine in the treatment of major depression. Psychiatric Services, 53, 585-590.

Churchill, R., Hunot, V., Corney, R., et al (2001) A systematic review of controlled trials of the effectiveness and costeffectiveness of brief psychological treatments for depression. Health Technology Assessment, 5, 1-173.

Cooper, P. J., Murray, L., Wilson, A., et al (2003) Controlled trial of the short- and long-term effect of psychological treatment of post-partum depression. 1: Impact on maternal mood. British Journal of Psychiatry, 182, 412-419.

Coryell, W., Akiskal, H., Leon, A. C., et al (1994) The time course of untreated major depression: uniformity across episodes and samples. American Journal of Psychiatry, 51, 405-410.

Crits-Christoph, P. (1992) The efficacy of brief dynamic psychotherapy: a meta-analysis. American Journal of Psychiatry, 159, 325-333.

Davanloo, H. (ed.) (1980) Basic Principles and Techniques in Shortterm Dynamic Psychotherapy. Spectrum.

de Jonghe, F., Hendriksen, M., van Aalst, G., et al (2004) Psychotherapy alone and combined with pharmacotherapy in the treatment of depression. British Journal of Psychiatry, 185, $37-45$.

Fonagy, P., Roth, A. \& Higgitt, A. (2005) Psychodynamic psychotherapies. Evidence-based practice and clinical wisdom. Bulletin of the Menninger Clinic, 69, 13. 
Freud, S. (1905) Three essays on the theory of sexuality. Reprinted (1953-1974) in the Standard Edition of the Complete Psychological Works of Sigmund Freud (trans. and ed. J. Strachey), vol. 7, pp. 125-243. Hogarth Press.

Freud, S. (1917) Mourning and melancholia. Reprinted (19531974) in the Standard Edition of the Complete Psychological Works of Sigmund Freud (trans. \& ed. J. Strachey), vol. 14, p. 237. Hogarth Press.

Goldberg, D. \& Goodyer, I. (2005) The Origins and Course of Common Mental Disorders. Routledge.

Goldberg, D., Privett, M., Ustun, B., et al (1998) The effects of detection and treatment on the outcome of major depression in primary care: a naturalistic study in 15 cities. British Journal of General Practice, 48, 1840-1844.

Hirschfeld, R. M. (1991) Depressive illness: diagnostic issues. Bulletin of the Menninger Clinic, 55, 144-155.

Judd, L. L. (1997) The clinical course of unipolar major depressive disorders. Archives of General Psychiatry, 54, 989991.

Judd, L. L., Akiskal, H. S. \& Paulus, M. P. (1997) The role and clinical significance of subsyndromal depressive symptoms (SSD) in unipolar major depressive disorder. Journal of Affective Disorders, 45, 5-17.

Kessler, R. C., Bergland, P., Demler, O., et al (2003) The epidemiology of major depressive disorder: results from the national comorbidity survey replication. JAMA, 289, 3095-3105.

Klein, M. (1940) Mourning and its relation to manic-depressive states. Reprinted (1975) in The Writings of Melanie Klein. Vol. 1 Love, Guilt and Reparation and Other Works 1921-1945. Hogarth Press.

Knekt, P., Lindfors, O., Harkanaen, T., et al (2008) Randomized trial on the effectiveness of long-and short-term psychodynamic psychotherapy and solution-focused therapy on psychiatric symptoms during a 3-year followup. Psychological Medicine, 38, 689-703.

Kopta, S., Howard, K., Lowry, J., et al (1994) Patterns of symptomatic recovery in psychotherapy. Journal of Consulting and Clinical Psychology, 62, 1009-1016.

Lambert, M. J. (1992) Psychotherapy outcome research: implications for integrative and eclectic theories. In Handbook of Psychotherapy Integration (eds J. C. Norcross \& M. R. Goldfried). Basic Books.

Lambert, M. J., Okiishi, J. C., Finch, A. E., et al (1998) Outcome assessment: from conceptualization to implementation. Professional Psychology: Research and Practice, 29, 63-70.

Lee, A. S. \& Murray, R. M. (1988) The long-term outcome of Maudsley depressives. British Journal of Psychiatry, 153, 741751.

Leichsenring, F. (2001) Comparative effects of short-term psychodynamic psychotherapy and cognitive-behavioural therapy in depression: a meta-analytic approach. Clinical Psychology Review, 21, 401-419.

Leichsenring, F., Rabung, S. \& Leibing, E. (2004) The efficacy of short-term psychodynamic psychotherapy in specific psychiatric disorders. A meta-analysis. Archives of General Psychiatry, 61, 1208-1216.

Lipsey, M.W. \&Wilson, D. B. (1993) The efficacy of psychological, education, and behavioral treatment: confirmation from meta-analysis. American Psychologist, 48, 1181-1209.

Luborsky, L., Singer B. \& Luborsky, L. (1975) Comparative studies of psychotherapies. Is it true that "everybody has won and all must have prizes"? Archives of General Psychiatry, 32, 995-1008.

Luborsky, L., Diguer, L., Seligman, D. A., et al (1999) The researcher's own therapy allegiances: a "wild card" in comparisons of treatment efficacy. Clinical Psychology: Science and Practice, 6, 95-106.

Malan, D. H. (1976) Toward the Validation of Dynamic Psychotherapy. Plenum Press.

Mueller, T. I., Leon, A. C., Keller, M. B., et al (1999) Recurrence after recovery from major depressive disorder during 15 years of observational follow-up. American Journal of Psychiatry, 156, 1000-1006.
McPherson, S., Cairns, P., Carlyle, J., et al (2005) The effectiveness of psychological treatments for treatment resistant depression: a systematic review. Acta Psychiatrica Scandinavica, 111, 331-340.

Milton, J. (2001) Psychoanalysis and cognitive behaviour therapy - rival paradigms or common ground? International Journal of Psychoanalysis, 82, 431-447.

National Collaborating Centre for Mental Health (2004) Depression: Management of Depression in Primary and Secondary Care. Clinical Guideline 23. NICE (http://guidance. nice.org.uk/CG23/?c=91523).

Paykel, E. S. (1994) Historical overview of outcome of depression. British Journal of Psychiatry, 165 (suppl. 26), 6-8.

Robins, L. N. \& Regier, D. A. (eds) (1991) Psychiatric Disorders in America: The Epidemiologic Catchment Area Study. The Free Press.

Robinson, L. A., Berma, J. S. \& Neimeyer, R. A. (1990) Psychotherapy for the treatment of depression: a comprehensive review of controlled outcome research. Psychological Bulletin, 108, 30-49.

Roth, A. \& Fonagy, P. (2004) What Works for Whom? A Critical Review of Psychotherapy Research (2nd edn). Guilford.

Sandell, R., Blomberg, J., Lazar, A., et al (2000) Varieties of longterm outcome among patients in psychoanalysis and longterm psychotherapy. A review of findings in the Stockholm Outcome of Psychoanalysis Project (STOPP). International Journal of Psychoanalysis, 81, 921-943.

Sargeant, J. K., Bruce, M. L., Florio, L. P., et al (1990) Factors associated with 1-year outcome of major depression in the community. Archives of General Psychiatry, 47, 519-526.

Shapiro, D. A., Barkham, M., Rees, A., et al (1994) Effects of treatment duration and severity of depression in the maintenance of gains following cognitive/behavioural and psychodynamic/interpersonal psychotherapy. Journal of Consulting and Clinical Psychology, 62, 522-534.

Spijker, J., de Graaf, R., Bijl, R. V., et al (2002) Duration of major depressive episodes in the general population: results from The Netherlands Mental Health Survey and Incidence Study (NEMESIS). British Journal of Psychiatry, 181, 208-213.

Stimpson, N., Agrawal, N. \& Lewis, G. (2002) Randomised controlled trials investigating pharmacological and psychological interventions for treatment-refractory depression. Systematic review. British Journal of Psychiatry, 181, 284-294.

Surtees, P. G. \& Barkley, C. (1994) Future imperfect: long-term outcome of depression. British Journal of Psychiatry, 164, 327-341.

Svartberg, M. \& Stiles, T. C. (1991) Comparative effects of short-term psychodynamic psychotherapy: a meta-analysis. Journal of Consulting and Clinical Psychology, 59, 704-714.

Thase, M. E., Greenhouse, J. B., Frank, E., et al (1997) Treatment of major depression with psychotherapy or psychotherapypharmacotherapy combinations. Archives of General Psychiatry, 54, 1009-1015.

Thompson, L. W., Gallagher, D. \& Breckenridge, J. S. (1987) Comparative effectiveness of psychotherapies for depressed elders. Journal of Consulting and Clinical Psychology, 55, 385390.

Wampold, B. E., Minami, T., Baskin, T. W., et al (2002) A meta(re)analysis of the effects of cognitive therapy versus 'other therapies' for depression. Journal of Affective Disorders, 68, 159-165.

Wampold, B. E., Mondin, G. W., Moody, M., et al (1997) A metaanalysis of outcome studies comparing bona fide psychotherapies: empirically, 'all must have prizes'. Psychological Bulletin, 122, 203-215.

Weissman, M. M., Bruce, M. B., Leaf, P. J. et al (1991) Affective disorders. In Psychiatric Disordersin America:The Epidemiologic Catchment Area Study (eds L. N. Robins \& D. A. Regier), pp. 53-80. The Free Press.

Westen, D., Novotny, C. M. \& Thompson-Brenner, H. (2004) The empirical status of empirically supported psychotherapies: assumptions, findings, and reporting in controlled clinical trials. Psychological Bulletin, 130, 631-663. 


\section{MCQs}

1 In individuals presenting with major depressive disorder:

a there is little likelihood of panic disorder

b full recovery is usual and recurrence unlikely

c asidefrom thesymptoms of depression the personality is usually normal

d personal loss plays no part

e there may be ongoing difficulties in personal functioning in periods of recovery.

2 According to the psychoanalytic theory of depression:

a thoughts and feelings outside core awareness play no part in the condition

$\mathrm{b}$ difficulties with anger and aggression are not found

c the concept of the 'object' refers to any inanimate thing

d emotional ambivalence deriving from early childhood relationships and losses is crucial

e the depressed person's superego is usually benign and forgiving.

3 With respect to the treatment of depression the evidence indicates that:

a short-term psychodynamic psychotherapy offers no benefit

b patient preference should not be taken into account

c on average, $\leq 20$ sessions of psychological therapy will move patients' Beck Depression Inventory scores into the normal range d longer-term therapies (e.g. 300 sessions) may offer benefits not possible with brief treatments

e the effects of short-term psychodynamic psychotherapy are usually evident immediately.

4 The evidence base with respect to the treatment of depression:

a offers clear indications about the best treatment of chronic or recurrent depression

$\mathrm{b}$ is derived from many good-quality RCTs with clinically representative samples and long followups

c is bedevilled by the effects of researcher allegiance and publication bias

$\mathrm{d}$ is of no value

$\mathrm{e}$ is very reassuring.

\section{MCQ answers}

$\begin{array}{llllll}1 & & 2 & & 3 & 4 \\ \text { a F } & \text { a F } & \text { a F } & \text { a F } \\ \text { b F } & \text { b F } & \text { b F } & \text { b F } \\ \text { c F } & \text { c F } & \text { c F } & \text { c T } \\ \text { d F } & \text { d T } & \text { d T } & \text { d F } \\ \text { e T } & \text { e F } & \text { e F } & \text { e F }\end{array}$

